\title{
High-dose MTX110 (soluble panobinostat) safely administered into the fourth ventricle in a nonhuman primate model
}

\author{
David I. Sandberg, MD, ${ }^{1,2}$ Natasha Kharas, BS, ${ }^{1,2}$ Bangning Yu, MD, PhD, ${ }^{1,2}$ \\ Christopher F. Janssen, DVM, ${ }^{3}$ Amanda Trimble, BS, ${ }^{3}$ Leomar Y. Ballester, MD, PhD, ${ }^{2,4}$ \\ Rajan Patel, MD, ${ }^{5}$ Afroz S. Mohammad, PhD, ${ }^{6}$ William F. Elmquist, PharmD, PhD, ${ }^{6}$ and \\ Rachael W. Sirianni, PhD²
}

\begin{abstract}
Departments of ${ }^{1}$ Pediatric Surgery and ${ }^{2}$ Neurosurgery, ${ }^{3}$ Center for Laboratory Animal Medicine and Care, and Departments of ${ }^{4}$ Pathology and Laboratory Medicine and ${ }^{5}$ Diagnostic and Interventional Imaging, McGovern Medical School, The University of Texas Health Science Center at Houston, Texas; and ${ }^{6}$ Department of Pharmaceutics, University of Minnesota, Minneapolis, Minnesota
\end{abstract}

OBJECTIVE Chemotherapy infusions directly into the fourth ventricle may play a role in treating malignant fourth-ventricular tumors. This study tested the safety and pharmacokinetics of short-term and long-term administration of MTX110 (soluble panobinostat; Midatech Pharma) into the fourth ventricle of nonhuman primates.

METHODS Four rhesus macaque monkeys underwent posterior fossa craniectomy and catheter insertion into the fourth ventricle. In group I $(n=2)$, catheters were externalized and lumbar drain catheters were placed simultaneously to assess CSF distribution after short-term infusions. MTX110 ( $0.5 \mathrm{ml}$ of $300 \mu \mathrm{M}$ panobinostat solution) was infused into the fourth ventricle daily for 5 consecutive days. Serial CSF and serum panobinostat levels were measured. In group II $(n=$ 2), fourth-ventricle catheters were connected to a subcutaneously placed port for subsequent long-term infusions. Four cycles of MTX110, each consisting of 5 daily infusions $(0.5 \mathrm{ml}$ of $300 \mu \mathrm{M}$ panobinostat solution), were administered over 8 weeks. Animals underwent detailed neurological evaluations, MRI scans, and postmortem histological analyses.

RESULTS No neurological deficits occurred after intraventricular MTX110 infusions. MRI scans showed catheter placement within the fourth ventricle in all 4 animals, with extension to the cerebral aqueduct in 1 animal and into the third ventricle in 1 animal. There were no MRI signal changes in the brainstem, cerebellum, or elsewhere in the brains of any of the animals. Histologically, normal brain cytoarchitecture was preserved with only focal mild postsurgical changes in all animals. Panobinostat was undetectable in serum samples collected 2 and 4 hours after infusions in all samples in both groups. In group I, the mean peak panobinostat level in the fourth-ventricle CSF (6242 ng/ml) was significantly higher than that in the lumbar CSF $(9 \mathrm{ng} / \mathrm{ml} ; \mathrm{p}<0.0001)$. In group II, the mean peak CSF panobinostat level $(11,042 \mathrm{ng} /$ $\mathrm{ml}$ ) was significantly higher than the mean trough CSF panobinostat level $(33 \mathrm{ng} / \mathrm{ml} ; \mathrm{p}<0.0001)$.

CONCLUSIONS MTX110 can be safely infused into the fourth ventricle in nonhuman primates at supratherapeutic doses. Postinfusion CSF panobinostat levels peak immediately in the fourth ventricle and then rapidly decrease over 24 hours. Panobinostat is detectable at low levels in CSF measured from the lumbar cistern up to 4 hours after infusions. These results will provide background data for a pilot clinical trial in patients with recurrent medulloblastoma. https://thejns.org/doi/abs/10.3171/2020.2.PEDS19786

KEYWORDS brain tumor; fourth ventricle; intraventricular; primate; panobinostat; medulloblastoma; oncology

$\mathrm{N}$ EW strategies are needed to treat medulloblastoma and other malignant posterior fossa tumors. Local delivery of chemotherapy into the fourth ventricle has potential advantages over systemic chemotherapy and other methods of intrathecal or intraventricular adminis- tration. Fourth-ventricle infusions yield high drug concentrations at the primary site of disease, where tumor is often left behind at the time of surgery because of its adherence to the brainstem. ${ }^{1-4}$ Because the fourth ventricle communicates both with other ventricular compartments

ABBREVIATIONS HDACi = histone deacetylase inhibitor; IM = intramuscular; MTX110 = soluble panobinostat.

SUBMITTED December 31, 2019. ACCEPTED February 27, 2020.

INCLUDE WHEN CITING Published online May 1, 2020; DOI: 10.3171/2020.2.PEDS19786. 
and with the spinal subarachnoid space, drugs infused into the fourth ventricle will distribute throughout the CSF-filled spaces of the brain and spine, thereby potentially treating both local and regional disease simultaneously. Unlike lateral ventricle catheters, which require a separate surgical procedure and blind catheter placement through brain parenchyma, fourth-ventricle catheters can be placed at the time of surgical resection of posterior fossa tumors without passing through any brain tissue. Placing fourth-ventricular catheters under direct vision should eliminate the risks of brain hemorrhage and catheter malposition associated with lateral-ventricle catheter placement. $^{5}$

Prior studies in our laboratory in piglets and nonhuman primates demonstrated that various chemotherapeutic agents can be safely infused into the fourth ventricle without causing neurological deficits or evidence of brain damage on MRI scans or histological analysis. ${ }^{1-3,6}$ In our prior studies, serum drug levels were negligible or undetectable, suggesting that systemic toxicity would be unlikely. High peak drug levels were observed in the fourth ventricle, and cytotoxic drug levels were sustained in lumbar CSF samples. Thus, high local and regional drug levels can potentially be achieved via fourth-ventricle infusions. These studies led to several pilot clinical trials in children with recurrent malignant brain tumors. ${ }^{7,8}$ These pilot trials confirmed the safety of this approach, with no new neurological deficits caused by fourth-ventricular chemotherapy infusions. Significantly, some patients exhibited decreased tumor size after treatment.

The objective of the current study was to evaluate the safety and pharmacokinetics of MTX110 infusion into the fourth ventricle. Panobinostat is a histone deacetylase inhibitor (HDACi) that decreases medulloblastoma tumor growth in orthotopic, patient-derived models. ${ }^{9}$ While panobinostat has been previously administered via convection-enhanced delivery into the brainstem in animals and is being administered in humans in an ongoing clinical trial (NCT03566199) ${ }_{1}^{10}$ there are to our knowledge no prior published reports describing intraventricular or intrathecal administration of panobinostat. Thus, novel aspects of the current experiments include drug delivery into the fourth ventricle, which has been reported in only a few prior studies, ${ }^{1-3,7,8}$ and intraventricular administration of panobinostat. The most logical potential application, if safety is demonstrated, is the use of MTX110 to treat recurrent medulloblastoma in the fourth ventricle and/or posterior fossa tumor resection cavity and potentially to treat recurrences in other intraventricular compartments or the leptomeninges.

\section{Methods}

\section{Experimental Groups}

All animal experimentation was conducted with the approval of the Animal Welfare Committee of the McGovern Medical School/University of Texas Health Science Center in Houston (protocol no. AWC-18-0074).

Four nonhuman primates (Chinese-origin rhesus macaques), 4-7 years old with weights of $6.3-8.4 \mathrm{~kg}$, were used. Two animals were male, and 2 were female. In group I (primates 1 and 2), to study short-term infusions of MTX110 into the fourth ventricle, a catheter was placed into the fourth ventricle and externalized for subsequent access. A lumbar drain catheter was placed simultaneously to study CSF pharmacokinetics. In group II (primates 3 and 4), to study long-term administration of MTX110 into the fourth ventricle, a catheter was inserted into the fourth ventricle and connected to a port placed subcutaneously for subsequent access.

\section{Anesthesia and Medications}

Anesthesia was induced using intramuscular (IM) midazolam $(0.1 \mathrm{mg} / \mathrm{kg})$ and ketamine $(10-25 \mathrm{mg} / \mathrm{kg})$ followed by endotracheal intubation and mechanical ventilation. Inhaled isoflurane was titrated to maintain anesthesia throughout the surgery. Heart rate and oxygen saturation were continuously monitored. At the conclusion of the surgery, isoflurane was stopped and animals were extubated following observation of spontaneous breathing with maintenance of normal oxygen saturations. Antibiotic prophylaxis with cefazolin $(55 \mathrm{mg} / \mathrm{kg} \mathrm{IM})$ was administered prior to skin incision and then daily for 5 days. Buprenorphine $(0.02-0.03 \mathrm{mg} / \mathrm{kg} \mathrm{IM})$ was administered twice daily for 3 days postoperatively for pain control. Maropitant (1 mg IM) was administered as needed postoperatively for nausea or vomiting.

\section{Surgical Procedures}

After intubation, animals were positioned prone with the head flexed. The skin was prepped and infiltrated with $0.5 \%$ bupivicaine hydrochloride. A midline posterior incision was made. After incision of the posterior cervical fascia and muscles, a limited suboccipital craniectomy and $\mathrm{C} 1$ laminectomy were performed. The dura mater was opened, and the inferior cerebellum was exposed and gently elevated to identify the floor of the fourth ventricle. A silicone lumbar drain catheter (Medtronic, product reference number 46419) was precut to a length of $16 \mathrm{~cm}$ and then placed under direct vision to ensure that all catheter holes were within the fourth ventricle. The dura mater was sutured closed around the catheter. Several pieces of pericranium were harvested from the skull and glued to the dura with n-butyl cyanoacrylate (3M Vetbond tissue adhesive) to prevent subsequent egress of CSF or infused MTX110. Several Valsalva maneuvers were performed to ensure that the closure was watertight. In group I, the catheter was externalized, attached to a Luer lock connector, and secured to the skin with sutures. In group II, the catheter was cut further, to the appropriate length to be connected to a port with a $0.05-\mathrm{ml}$ volume (Access Technologies, item number CP6AC-5NC) that was placed subcutaneously inferior to the incision for subsequent access. In both groups, the muscle, fascia, and skin were closed in a routine fashion.

In addition to fourth-ventricular catheter placement, animals in group I also underwent simultaneous placement of an externalized lumbar drain catheter. A separate incision in the lumbar spine region was made after skin infiltration with $0.5 \%$ bupivicaine hydrochloride. A limited laminectomy and small durotomy were performed, 
and a silicone lumbar drain catheter (Medtronic, product reference number 46419) precut to a length of $16 \mathrm{~cm}$ was inserted rostrally into the subdural space. The dura was sutured closed around the catheter, and a watertight dural closure was ensured by securing several pieces of lumbar fascia to the dura with n-butyl cyanoacrylate (3M Vetbond tissue adhesive). Several Valsalva maneuvers were performed to demonstrate a lack of CSF egress. The catheter was externalized, attached to a Luer lock connector, and secured to the skin with sutures. The muscle, fascia, and skin were closed in routine fashion.

At the conclusion of the surgery, prior to extubation, a primate jacket was used to protect the externalized drains (group I) or subcutaneous port (group II).

\section{Clinical Neurological Assessment}

All animals were examined at least once daily by a veterinary technician and at least twice weekly by a veterinarian throughout the experiment to assess for morbidity. In addition, a detailed neurological examination scored using a grading scale developed at our institution (Table 1) was performed at set time points. For the group I primates, a detailed neurological examination was performed preoperatively, postoperatively prior to infusions, after the first infusion, and after the final infusion. For the group II primates, a detailed neurological examination was performed preoperatively, postoperatively prior to infusions, and after completion of each cycle of MTX110 infusions (4 additional times). For each neurological examination, a score was assigned by at least 2 observers, at least one of whom was a veterinarian or veterinary technician. Preexperiment scores were compared to postinfusion scores to assess for new neurological deficits caused by surgery and/ or MTX110 infusions.

\section{MTX110 Infusions and CSF and Serum Analysis}

To guide the selection of an appropriate dose for nonhuman primate experiments, we first studied intracisternal infusion of MTX110 in C57bl/6 mice. Peak weight loss percentages observed 2 days after a $2-\mu 1$ infusion of 90 , 300 , or $800 \mu \mathrm{M}$ panobinostat were observed to be $4.25 \%$ $\pm 2.2 \%, 7.85 \% \pm 4.3 \%$, and $10.8 \% \pm 0.68 \%$, respectively (n $=3-4$ mice per group). The midrange dose of $300 \mu \mathrm{M}$ was selected for primate studies, with scaling of $2 \mu \mathrm{l}$ to $0.5 \mathrm{ml}$ based on the expected CSF volume of a mouse versus a rhesus macaque (40 $\mu \mathrm{l}$ to $10 \mathrm{ml}$ ).

For MTX110 infusions and CSF and serum sampling, primates were sedated with ketamine ( 7.3 to $22 \mathrm{mg} / \mathrm{kg} \mathrm{IM}$ ) and positioned on a procedure table. MTX110 (Midatech Ltd.) was diluted in sterile preservative-free normal saline to a concentration of $300 \mu \mathrm{M}$ in $0.5 \mathrm{ml}$ total volume. Each MTX110 dose was infused into the fourth ventricular catheter over a 3-minute period. For group I primates, immediately after each infusion, $0.2 \mathrm{ml}$ of sterile preservative-free normal saline was infused over 1 minute to ensure that all of the drug was flushed out of the tubing and into the fourth ventricle. This volume was determined based on intraoperative testing that demonstrated a catheter dead space of less than $0.15 \mathrm{ml}$. For group II primates, $0.3 \mathrm{ml}$ of sterile preservative-free normal saline was in- fused over 1 minute to account for the dead space in the tubing and the port.

For group I primates, MTX110 infusions into the externalized fourth-ventricle catheter were performed once daily for 5 consecutive days beginning at least 3 days postoperatively. CSF samples $(0.5 \mathrm{ml})$ were obtained simultaneously from the fourth ventricle and lumbar catheters at 15 minutes and then $1,2,4,8,12$, and 24 hours after the first infusion. Prior to CSF sampling, $0.2 \mathrm{ml}$ of CSF was aspirated and discarded from externalized catheters to account for dead space. After CSF sampling, each catheter was flushed with $0.7 \mathrm{ml}$ of preservative-free normal saline. For the next 4 days, CSF samples were obtained immediately before and 15 minutes after each subsequent MTX110 infusion to monitor trough and peak levels. Serum samples ( $3 \mathrm{ml}$ collected blood) were obtained via femoral vein puncture 2 and 4 hours after the first infusion.

For group II primates, MTX110 infusions were administered via a Tuohy needle inserted into the subcutaneous port. Peak and trough CSF panobinostat levels $(0.5 \mathrm{ml})$ were obtained on each day of MTX110 administration. Prior to CSF sampling, $0.3 \mathrm{ml} \mathrm{CSF}$ was aspirated and discarded from externalized catheters to account for dead space. After CSF sampling, each catheter was flushed with $0.7 \mathrm{ml}$ preservative-free normal saline. Weeks that included infusions alternated with weeks of observation. Four cycles of intraventricular MTX110 infusions were administered over 8 weeks, with each cycle consisting of 5 consecutive daily infusions. Serum samples $(3 \mathrm{ml} \mathrm{col}-$ lected blood) were obtained via femoral vein puncture 2 and 4 hours after the first intraventricular MTX110 dosing for each cycle.

After collection, CSF and serum samples were centrifuged and stored at $-80^{\circ} \mathrm{C}$. Panobinostat levels were determined using standardized liquid chromatography with tandem mass spectrometry by investigators blinded to the timing of CSF and serum sampling. All samples were spiked with panobinostat-d8 hydrochloride salt as the internal standard. After extraction in ethyl acetate, the organic supernatant was dried under nitrogen, reconstituted in mobile phase (80:20 distilled water with $0.1 \%$ formic acid:acetonitrile with $0.1 \%$ formic acid), and injected onto a YMC-pack ODS-AM column $(35 / 2.0 \mathrm{~mm}, \mathrm{~S}-3 \mu \mathrm{m}, 12$ $\mathrm{nm}$; YMC, Inc.) for liquid chromatography. The column effluent was monitored using a Micromass Quattro Ultima mass spectrometer (Waters). The mass to charge ratios (m/z transitions) were 350.04-157.91 for panobinostat and 357.92-165.04 for panobinostat-d8 hydrochloride salt (positive-ionization mode). The LOQ (limit of quantification) was $1 \mathrm{ng} / \mathrm{mL}$ and the precision (expressed as the CV percentage) was less than $15 \%$. The statistical significance of the differences between the fourth-ventricle and lumbar CSF panobinostat levels and between the peak and trough fourth-ventricle CSF panobinostat levels was assessed by t-tests at a 5\% significance level, utilizing paired t-tests where appropriate.

\section{MRI Scans}

MRI scans were performed on a 3.0-Tesla MRI magnet after completion of the final infusion. T2-weighted axial, coronal, and sagittal views were obtained to assess the 
TABLE 1. Primate neurological examination grading scale

\begin{tabular}{|c|c|}
\hline Scoring Category \& No. of Points & Observed Behavior \\
\hline \multicolumn{2}{|l|}{ Level of consciousness } \\
\hline 0 & Very low arousal state (unconscious, no response to observer or physical stimuli) \\
\hline 1 & $\begin{array}{l}\text { Low arousal state (no activity, does not respond to presence of observer, responds } \\
\text { to physical stimuli only) }\end{array}$ \\
\hline 2 & $\begin{array}{l}\text { Normal, quiet, or agitated (directs attention to the presence of the observer, moves } \\
\text { calmly or energetically around cage) }\end{array}$ \\
\hline \multicolumn{2}{|l|}{ Cranial nerve examination } \\
\hline \multicolumn{2}{|l|}{ Extraocular movement } \\
\hline 0 & No eye movement in any direction \\
\hline 1 & Limitation in eye movements in 1 or both eyes in 1 or more direction \\
\hline 2 & Eye movement in each direction but eyes are dysconjugate \\
\hline 3 & Normal extraocular movements \\
\hline \multicolumn{2}{|l|}{ Facial muscle movement } \\
\hline 0 & No movement of facial muscles on either side \& cannot close either eye completely \\
\hline 1 & Paralysis or paresis of facial muscles on 1 side \& cannot close eye completely \\
\hline 2 & Paralysis or paresis of facial muscles on 1 side but can close eye completely \\
\hline 3 & Normal movement of facial muscles bilaterally \& closes both eyes completely \\
\hline \multicolumn{2}{|l|}{ Auditory response } \\
\hline 0 & No response to auditory threat (clapping hands or other noise) \\
\hline 1 & Response to noise is evident \\
\hline \multicolumn{2}{|l|}{ Lower cranial nerves assessed by ability to swallow } \\
\hline 0 & Choking on food or water, difficulty swallowing solids or liquid \\
\hline 1 & Not choking on food or water, no difficulty swallowing solids or liquids \\
\hline \multicolumn{2}{|l|}{ Motor, coordination (cerebellar), \& gait examinations } \\
\hline \multicolumn{2}{|c|}{$\begin{array}{l}\text { Motor examination (each of all } 4 \text { extremities, maximum }=8 \\
\text { points) }\end{array}$} \\
\hline 0 & No movement \\
\hline 1 & Some movement but paretic \\
\hline 2 & Normal movement \\
\hline \multicolumn{2}{|c|}{$\begin{array}{l}\text { Coordination (cerebellar) assessed by observation of reaching } \\
\text { for objects such as food ( } 2 \text { upper extremities, maximum }=2 \\
\text { points) }\end{array}$} \\
\hline 0 & Uncoordinated/dysmetria when reaching for objects \\
\hline 1 & Normal coordination/no dysmetria when reaching for objects \\
\hline \multicolumn{2}{|c|}{ Gait examination (observed in cage \& walk w/ pole \& collar) } \\
\hline 0 & Not ambulatory \\
\hline 1 & $\begin{array}{l}\text { Ambulatory but abnormal gait pattern (difficulty maintaining balance, limping, or } \\
\text { other abnormal gait pattern) }\end{array}$ \\
\hline 2 & Normal gait pattern (balanced, well-articulated climbing \& walking) \\
\hline \multicolumn{2}{|l|}{ Maximum total points } \\
\hline 22 & \\
\hline
\end{tabular}

catheter position within the fourth ventricle and to look for any brain abnormalities. FLAIR sequences were performed in the axial, coronal, and sagittal planes to detect transependymal fluid absorption and other brain lesions. Axial diffusion-weighted sequences, including ADC (apparent diffusion coefficient) maps were obtained to detect ischemic changes. MRI scans were reviewed independently by a neuroradiologist.

\section{Postmortem Histological Analysis}

After the MRI scans were completed, while the animals were still under isoflurane anesthesia, they were transported to the necropsy room. There, the isoflurane concentration was increased to $5 \%$. After deep anesthesia was confirmed by the absence of all reflexes, the heart was exposed through intercostal thoracotomy and transverse sternotomy. A 14-gauge ball-tipped feeding needle was 

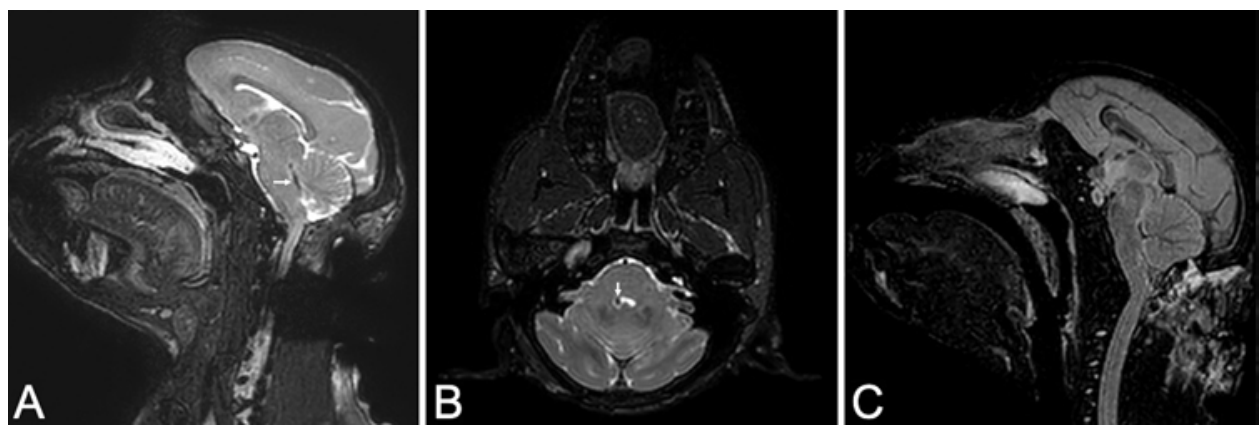

FIG. 1. MRI scans obtained after MTX110 infusions. A: Sagittal T2-weighted MRI in primate 1. The white arrow points to the catheter within the fourth ventricle. B: Axial T2-weighted MRI in primate 1. The white arrow points to the catheter within the fourth ventricle. C: Sagittal FLAIR MRI in primate 3. No abnormal signal changes are noted in the brainstem, cerebellum, or elsewhere after infusions.

introduced into the apex of the left ventricle, and the apex was clamped to prevent leakage. The right auricle was perforated to allow euthanasia by exsanguination and perfusion. As venous blood was drained from the right auricle, $1 \mathrm{~L}$ of $0.9 \%$ saline was administered into the left ventricle under pressure, followed by $1 \mathrm{~L}$ of $10 \%$ neutral buffered formalin. The brain was removed, taking care to examine the catheter location. The spinal cord was then harvested. Representative samples of the dura, liver, spleen, lung, heart, kidney, and mesenteric lymph nodes were collected. Specimens were placed in $10 \%$ neutral buffered formalin for at least 1 week prior to cutting. Specimens were sectioned, embedded in paraffin, cut, and stained with $\mathrm{H}$ \& E for subsequent microscopic examination by a neuropathologist.

\section{Results}

\section{Clinical Findings}

No neurological deficits were caused by MTX110 infusions in any animals. Additionally, no changes in feeding patterns or other systemic concerns were noted by veterinarians or veterinary technicians. No primates were noted to have a pseudomeningocele or CSF leak. In both primates in group I, the maximum number of points on the detailed neurological examination (22) was achieved at every time point. In group II, both primates scored 21 of 22 maximum points preoperatively, losing 1 point because of the lack of auditory threat response. The first animal in group II, primate number 3 , scored 15 out of 22 points postoperatively (before any MTX110 infusions) due to decreased coordination and possible weakness of each limb, right worse than left, and altered gait. This score gradually improved to the preoperative baseline ( 21 out of 22 points) by the end of the 2 nd week of infusions, and this score was maintained for the remainder of the experiment. The second animal in group II, primate number 4 , had partial wound dehiscence noted several days postoperatively, and her fourth-ventricle catheter could not reliably aspirate CSF. She was therefore taken back to surgery for both wound and catheter revisions. At surgery, the ventricular catheter was removed and was found to have debris in all catheter holes. A new catheter was placed under direct vision into the fourth ventricle and attached to the existing ventricular access device. The remainder of the surgical procedure was performed as described in the Methods section above. This animal scored $21 / 22$ points on the neurological examination performed in all preoperative, postoperative, and postinfusion assessments.

\section{Imaging Findings}

T2-weighted MRI sequences demonstrated catheter placement within the fourth ventricle in all animals (Fig. $1 \mathrm{~A}$ and $\mathrm{B}$ ). In 2 animals, the catheter tip terminated within the fourth ventricle. In 1 animal in each group, the catheter tip terminated beyond the fourth ventricle (at the level of the cerebral aqueduct in primate 2 and within the third ventricle in primate 4). Ventricular size was normal in all animals, indicating that catheter placement and panobinostat infusion did not cause hydrocephalus. In animals from both groups, FLAIR and diffusion-weighted sequences did not demonstrate any evidence of ischemia or other abnormal signal changes in the brainstem, cerebellum, or elsewhere in the brain (Fig. 1C).

\section{Histological Analysis}

Grossly, the brain and all other organs appeared to be normal upon visual inspection in all four primates. In all animals, when the brain was harvested, the ventricular catheter was visualized within the fourth ventricle with all catheter holes rostral to the obex (Fig. 2). Histological analysis of brain sections demonstrated preservation of the normal cytoarchitecture of all regions of the brain. There was no axonal degeneration or necrosis observed. A tiny area of mild focal ependymal disruption in the pons was visualized in both primates in group I and primate 4 in group II (Fig. 3). This finding was likely a postsurgical change resulting from catheter placement. In all animals, mild focal inflammatory infiltrates were observed in the leptomeninges, choroid plexus, or focally in the subependymal zone of the brainstem (Fig. 3). Histological analysis of the spinal cord, dura, liver, spleen, lung, heart, kidney, and mesenteric lymph nodes showed no evidence of systemic toxicity from fourth-ventricular infusions.

\section{Pharmacokinetic Analysis}

Panobinostat was undetectable in all serum samples 


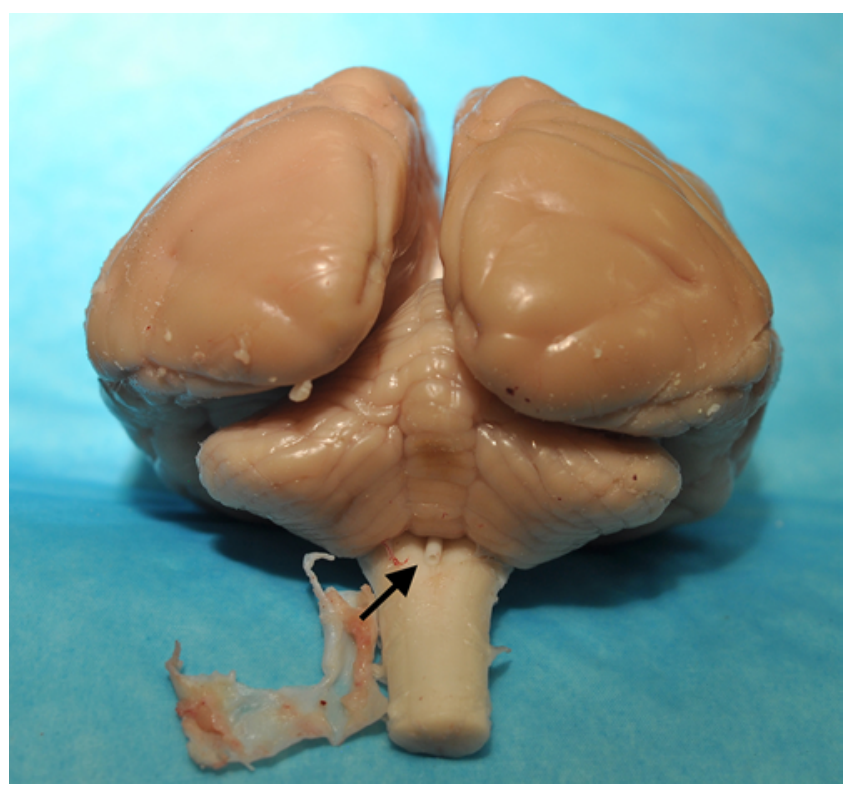

FIG. 2. Brain harvested from primate 2 after euthanasia. The brain appears grossly normal. The catheter, as noted by the black arrow, is within the fourth ventricle. No catheter holes are visualized because they are all above the level of the obex. Figure is available in color online only. collected at 2 and 4 hours postinfusion. For group I animals, postinfusion panobinostat levels peaked immediately and then steadily decreased over the first 24 hours in CSF from both the fourth ventricle and the lumbar cistern (Fig. 4). Panobinostat reached undetectable levels more rapidly in lumbar CSF than fourth-ventricle CSF. Panobinostat was undetectable in lumbar CSF after 2 hours postinfusion in primate 1 and after 4 hours postinfusion in primate 2 . In primate 2 , the lumbar catheter stopped working after 8 hours following the first infusion, so CSF could not be sampled subsequently. In CSF sampled from the fourth ventricle, panobinostat was still detected 24 hours after MTX110 infusion in 6 of 8 samples (Figs. 4 and 5). In both group I animals, the mean fourth-ventricle peak CSF panobinostat level $(6242 \mathrm{ng} / \mathrm{ml})$ exceeded the mean peak lumbar panobinostat level $(9 \mathrm{ng} / \mathrm{ml})$ by greater than 600 -fold. The highest measured lumbar level was $88 \mathrm{ng} / \mathrm{ml}$ (1 hour after infusion), and panobinostat was not detected in lumbar samples at most time points. For group I, the mean peak panobinostat level in the fourth-ventricle CSF was $6242 \mathrm{ng} / \mathrm{ml}$ (range 5057-7319 $\mathrm{ng} / \mathrm{ml}$ ), and the mean trough panobinostat level in the fourth-ventricle CSF was $34 \mathrm{ng} / \mathrm{ml}$ (range 0-71 ng/ml) (Fig. 5). Peak panobinostat CSF levels were significantly higher in the fourth ventricle than the lumbar cistern $(\mathrm{p}<0.0001)$. Panobinostat levels were undetectable at troughs in lumbar CSF. Thus, trough

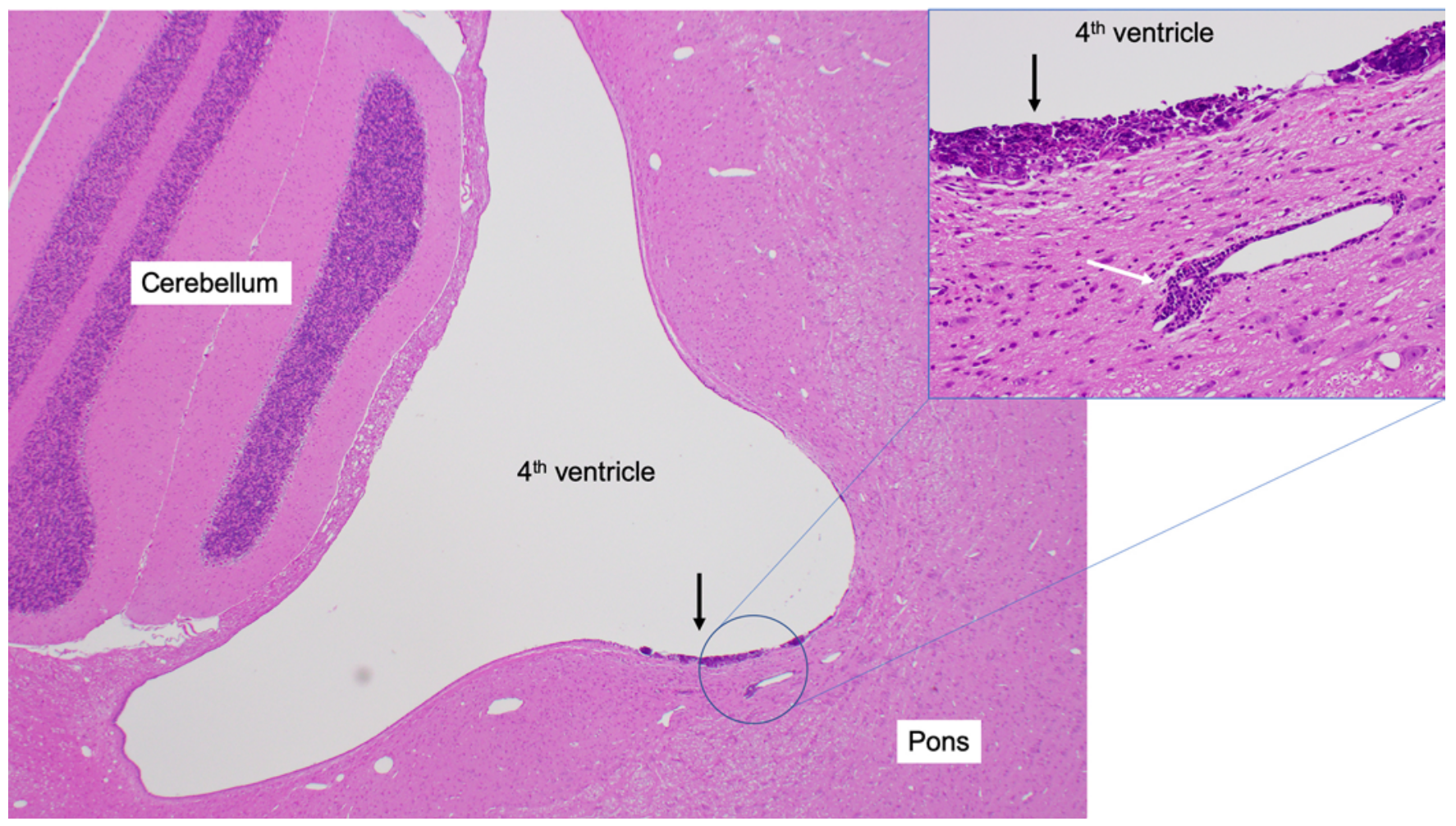

FIG. 3. H \& E-stained section showing the cerebellum, fourth ventricle, and pons from primate 1. Inset: A higher-magnification image of the region within the blue circle in the tegmentum, showing focal disruption of the ependymal lining (black arrow) and focal perivascular inflammation (white arrow). 


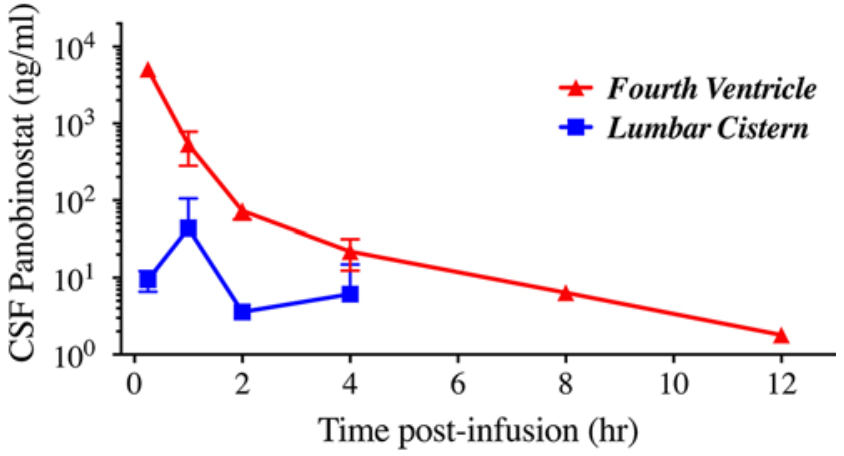

FIG. 4. Graph of panobinostat levels in CSF sampled from the fourth ventricle and the lumbar cistern over the first 12 hours postinfusion into the fourth ventricle in group I animals. Panobinostat levels 24 hours postinfusion were undetectable in either location. Data points represent the mean for $n=2(0.25,1,2,4$, and 8 hours) or $n=1$ (12 hours) for fourth-ventricle samples and $n=2(0.25,1$, and 4 hours) or $n=1$ (2 hours) for lumbar cistern samples. Error bars represent standard deviations. Figure is available in color online only.

panobinostat CSF levels were also significantly higher in the fourth ventricle than the lumbar cistern $(\mathrm{p}<0.0001)$. When the peak and trough analyses for group I and II were combined (Fig. 6), the peak fourth-ventricle CSF panobinostat levels ranged from 4547 to $18,188 \mathrm{ng} / \mathrm{ml}$ (mean 9671 $\pm 3577 \mathrm{ng} / \mathrm{ml}$ for 35 measured samples). The trough fourthventricle CSF panobinostat levels ranged from below the lower limit of quantification to $121.3 \mathrm{ng} / \mathrm{ml}$ (mean $29 \pm$ $24 \mathrm{ng} / \mathrm{ml}$ for 35 measured samples). Peak fourth-ventricle CSF levels were significantly higher than trough fourthventricle CSF levels in all cycles in both animals in groups I and II $(\mathrm{p}<0.0001)$.

\section{Discussion}

This study describes what is to our knowledge the first reported intraventricular administration of MTX110, a soluble form of panobinostat, in an animal model. Panobinostat is a chemotherapeutic agent that is promising for the treatment of medulloblastoma. In 2016, Pei et al. reported that panobinostat was the most promising HDACi among those identified in a high-throughput drug screen and tested on a genetically engineered mouse model of group 3 medulloblastoma. ${ }^{9}$ Panobinostat was also the most effective HDACi against a human group 3 medulloblastoma patient-derived xenograft (PDX) model. Panobinostat was also effective against PDX models of sonic-hedgehog and group 4 models in these studies. ${ }^{9}$ Thus, we hypothesize that this treatment approach may be most useful for treating group 3 medulloblastoma but may have treatment responses in other subgroups as well.

A primary objective of this study was to demonstrate that MTX110 may be safely infused into the fourth ventricle in nonhuman primates. Of note, the study was performed in rhesus monkeys that did not harbor brain tumors. There is no adequate large-animal model of medulloblastoma, and these experiments would be technically challenging, if not impossible, in small-animal models.

In this study, the safety of MTX110 infusions into the

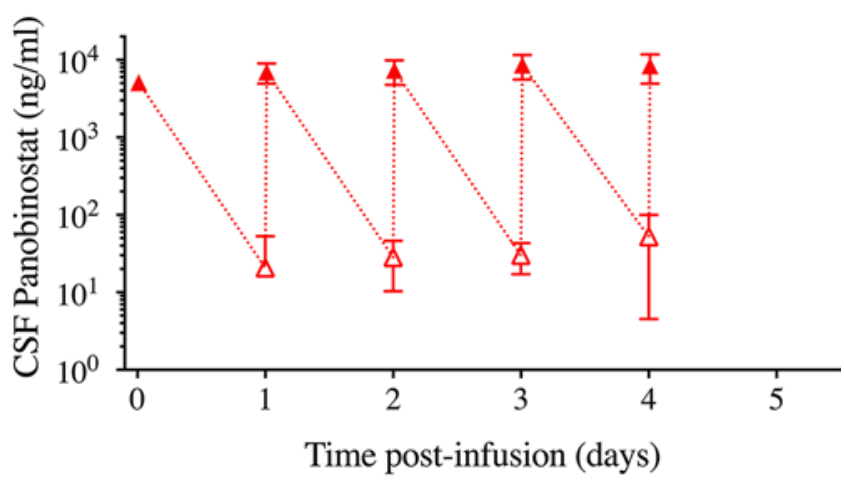

FIG. 5. Mean CSF panobinostat levels at peaks and troughs over 5 days of daily infusion into the fourth ventricle in group I and II animals. Data points represent the mean for $n=4$ at all time points. Error bars represent standard deviations. Figure is available in color online only.

fourth ventricle was demonstrated based on clinical neurological examinations, postinfusion MRI scans, and postmortem pathological analyses. In detailed neurological assessment after infusions, no new neurological deficits were noted in the primates. While 1 primate was worse neurologically postoperatively (prior to infusions) than preoperatively, her neurological deficits resolved completely while receiving infusions. The likely cause of this animal's postoperative neurological worsening was manipulation of the brain during catheter placement. These surgeries are technically more challenging in nonhuman primates than in humans, as the fourth ventricle in primates is smaller than in humans whose fourth ventricle has been expanded by a tumor. New neurological deficits after fourth-ventricular catheter placement have not occurred in human clinical trials of fourth-ventricular chemotherapy infusions to date. ${ }^{7,8}$ Because no validated neurological scoring scale for nonhuman primates has been reported in previous publications, we developed a primate neurological examination scoring scale at our own institution (Table 1) that was designed to detect the most likely expected neurological deficits from infusions into the fourth ventricle (e.g., cranial neuropathies, motor deficits, and cerebellar findings). In

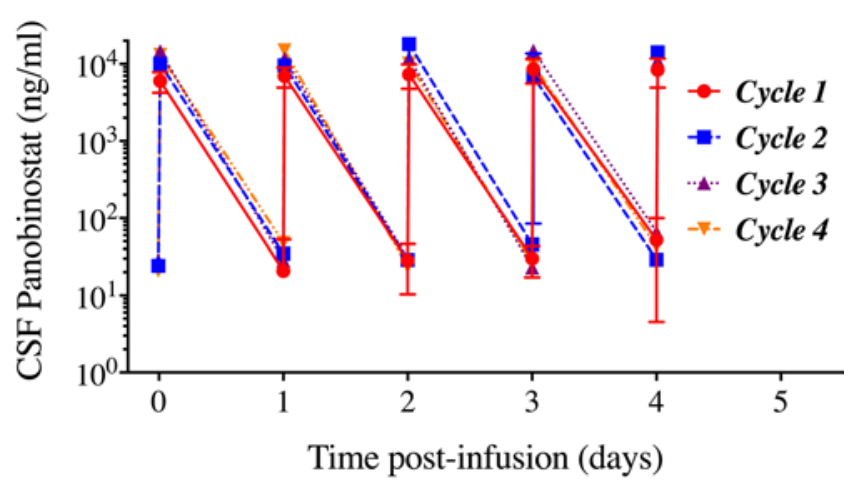

FIG. 6. Mean CSF panobinostat levels at peaks and troughs over 4 cycles of daily infusion into the fourth ventricle in group II animals. Data points represent the mean for $n=4$ in cycle 1 and $n=2$ in all other cycles. Error bars represent standard deviations. 
future studies, these preliminary findings could be investigated further with detailed functional analyses.

MRI scans demonstrated catheter placement within the fourth ventricle. In 2 of 4 animals, the catheter passed through the fourth ventricle and ended slightly more rostral than intended. This was due to the technical difficulties of keeping the catheter in a perfect position in the small fourth ventricle of a primate. In clinical trials to date performed in humans, in whom the fourth ventricle is larger, all prior catheter placements have been accurate, with catheter tips terminating within the fourth ventricle. ${ }^{7,8}$ Of note, in the current experiments, all catheters were found on postmortem exams to have entered the fourth ventricle, with no catheter holes visualized distal to the obex. Since the catheters have multiple holes coursing along their distal portion, we can conclude that all 4 primates received MTX110 into the fourth ventricle and that 2 of them additionally received MTX110 in the cerebral aqueduct and/or third ventricle. No animals had any evidence of ischemia or other damage to the brain after fourth-ventricle MTX110 infusions. The safety of MTX110 infusions into the fourth ventricle was also supported by postmortem histological evaluations. All brains and other evaluated organs looked grossly normal, and histological evaluation demonstrated only minor postoperative changes with no damage to the brain or any other organs.

Our analysis of serum and CSF panobinostat levels suggested several possible advantages to direct infusion of panobinostat into the fourth ventricle compared with systemic infusions. First, panobinostat was undetectable in serum. While a drug infused into any CSF compartment should eventually enter the bloodstream via normal CSF distribution pathways, the amount of panobinostat that entered the bloodstream was too low to be measured. The lack of systemic drug exposure suggests that systemic toxicity would be less likely with fourth-ventricular administration than with systemic administration. Moreover, since medulloblastoma and other malignant fourth-ventricular tumors in children only rarely metastasize outside of the CNS, high systemic drug levels may not be necessary if drug levels within circulating CSF spaces (where tumors originate and most often recur) are high.

An additional possible advantage of panobinostat infusions into the fourth ventricle over systemic infusions is the higher drug levels that can be achieved with fourthventricle infusions. In the current experiments, peak measurements in fourth-ventricle CSF were extremely high (mean $6242 \mathrm{ng} / \mathrm{ml}$ in group I and 11,042 $\mathrm{ng} / \mathrm{ml}$ in group II). By comparison, in a recent review article describing panobinostat pharmacokinetics, pooled data of multidose studies showed a maximum plasma concentration of 21.6 $\mathrm{ng} / \mathrm{ml} 1$ hour after administration of $20 \mathrm{mg}$ of oral panobinostat. ${ }^{11}$ In another recent publication, oral panobinostat $(60 \mathrm{mg})$ resulted in a maximum plasma concentration of $71 \mathrm{ng} / \mathrm{ml}$, while intravenous panobinostat at the highest dose administered $\left(14 \mathrm{mg} / \mathrm{m}^{2}\right)$ resulted in an estimated maximum plasma concentration of $565.5 \mathrm{ng} / \mathrm{ml} .{ }^{12} \mathrm{In}$ a prior study describing oral administration $(20 \mathrm{mg} 3$ times per week, every other week for 8 weeks), panobinostat was not measurable in CSF, suggesting that oral administration would likely be ineffective against CNS tumors. ${ }^{11}$ Despite the extremely high CSF panobinostat concentrations in the primates in our study, none showed any neurological impairment or evidence of damage to the brain on imaging studies or histological analysis. Because panobinostat administration by this method has not yet been performed in humans, the significance of these high concentrations with regard to reducing tumor cell burden is uncertain.

After reaching high peak panobinostat levels in fourthventricular CSF, panobinostat levels rapidly declined over the first 24 hours after infusion. Following subsequent infusions, trough levels were elevated relative to the first infusion, which could suggest a saturable clearance mechanism. At 24-hour trough measurements, panobinostat was measurable, albeit at low concentrations, in the majority of fourth-ventricle CSF samples. Analysis of lumbar CSF levels was limited in this study by the fact that 1 of the 2 lumbar catheters stopped working after 8 hours. Panobinostat was measurable at low concentration in lumbar CSF up to 4 hours after infusion, with no measurable panobinostat thereafter. By comparison, in similar prior experiments with a different agent, methotrexate, higher sustained lumbar CSF drug levels were measurable 24 hours after fourthventricle infusions. ${ }^{3}$ The rapid decline in measurable panobinostat in lumbar CSF in the current experiments suggests that infusions of MTX110 into the fourth ventricle may yield higher drug exposure to residual or recurrent tumor in the fourth ventricle than to leptomeningeal disease that has spread to the spine. The presence of measurable panobinostat in some lumbar cistern CSF samples suggests that the exposure of leptomeningeal disease to the drug is at least possible by this method unless there is a disturbance of CSF circulation caused by bulky leptomeningeal disease or scar tissue from prior treatments. It is our hypothesis that within the posterior fossa, smaller tumor volumes will be more effectively treated by this method than larger ones, and our plan for subsequent clinical trials is to debulk as much local disease as possible before implanting a ventricular access device and beginning infusions.

\section{Conclusions}

This study demonstrates that MTX110 (soluble panobinostat) can be safely infused into the fourth ventricle in nonhuman primates without causing neurological deficits, abnormalities visible on brain MRI, or pathological evidence of brain or other organ injury. Panobinostat is not detected in serum after these infusions, suggesting that systemic side effects would be unlikely with this treatment approach. High drug levels are achieved in the fourth ventricle and then quickly decline, but panobinostat is still measurable in the fourth-ventricle CSF 24 hours after infusions. Significantly lower drug levels are present for up to 4 hours in lumbar CSF. Direct infusion of MTX110 into the fourth ventricle is worthy of further investigation to determine if this approach may benefit patients with medulloblastoma or other malignant tumors originating or recurring in the fourth ventricle.

\section{Acknowledgments}

This work was supported by the Dr. Marnie Rose Foundation (David I. Sandberg, MD) and by a generous donation from 
Mr. Dick Bassett (David I. Sandberg, MD). We also gratefully acknowledge funding from the Eunice Kennedy Shriver National Institute of Child Health and Human Development (R01HD099543; Rachael W. Siranni, PhD, and David I. Sandberg, MD).

\section{References}

1. Sandberg DI, Crandall KM, Koru-Sengul T, et al. Pharmacokinetic analysis of etoposide distribution after administration directly into the fourth ventricle in a piglet model. $\mathrm{J} \mathrm{Neu}$ rooncol. 2010;97(1):25-32.

2. Sandberg DI, Crandall KM, Petito CK, et al. Chemotherapy administration directly into the fourth ventricle in a new piglet model. J Neurosurg Pediatr. 2008;1(5):373-380.

3. Sandberg DI, Peet MM, Johnson MD, et al. Chemotherapy administration directly into the fourth ventricle in a nonhuman primate model. J Neurosurg Pediatr. 2012;9(5):530-541.

4. Sandberg DI, Solano J, Petito CK, et al. Safety and pharmacokinetic analysis of methotrexate administered directly into the fourth ventricle in a piglet model. J Neurooncol. 2010;100(3):397-406.

5. Sandberg DI, Kerr ML. Ventricular access device placement in the fourth ventricle to treat malignant fourth ventricle brain tumors: technical note. Childs Nerv Syst. 2016;32(4):703-707.

6. Sandberg DI, Crandall K, Petito CK, et al. Distribution of etoposide in cerebrospinal fluid after infusions into the fourth ventricle in piglets. J Neurosurg Pediatr. 2008;1:A354.

7. Sandberg DI, Rytting M, Zaky W, et al. Methotrexate administration directly into the fourth ventricle in children with malignant fourth ventricular brain tumors: a pilot clinical trial. J Neurooncol. 2015;125(1):133-141.

8. Sandberg DI, Yu B, Patel R, et al. Infusion of 5-azacytidine (5-AZA) into the fourth ventricle or resection cavity in children with recurrent posterior fossa ependymoma: a pilot clinical trial. J Neurooncol. 2019;141(2):449-457.

9. Pei Y, Liu KW, Wang J, et al. HDAC and PI3K Antagonists cooperate to inhibit growth of MYC-driven medulloblastoma. Cancer Cell. 2016;29(3):311-323.
10. Singleton WGB, Bienemann AS, Woolley M, et al. The distribution, clearance, and brainstem toxicity of panobinostat administered by convection-enhanced delivery. $J$ Neurosurg Pediatr. 2018;22(3):288-296.

11. Van Veggel M, Westerman E, Hamberg P. Clinical pharmacokinetics and pharmacodynamics of panobinostat. Clin Pharmacokinet. 2018;57(1):21-29.

12. Savelieva M, Woo MM, Schran H, et al. Population pharmacokinetics of intravenous and oral panobinostat in patients with hematologic and solid tumors. Eur J Clin Pharmacol. 2015;71(6):663-672.

\section{Disclosures}

The authors report no conflict of interest concerning the materials or methods used in this study or the findings specified in this paper.

\section{Author Contributions}

Conception and design: Sandberg, Janssen. Acquisition of data: Sandberg, Kharas, Yu, Janssen, Trimble, Mohammad, Elmquist. Analysis and interpretation of data: Sandberg, Kharas, Janssen, Ballester, Patel, Mohammad, Elmquist, Sirianni. Drafting the article: Sandberg. Critically revising the article: Sandberg, Kharas, Janssen, Ballester, Patel, Mohammad, Elmquist, Sirianni. Reviewed submitted version of manuscript: all authors. Approved the final version of the manuscript on behalf of all authors: Sandberg. Statistical analysis: Kharas, Sirianni. Administrative/ technical/material support: Yu, Trimble. Study supervision: Sandberg.

\section{Correspondence}

David I. Sandberg: McGovern Medical School/University of Texas Health Science Center at Houston, Houston, TX. david.i.sandberg@uth.tmc.edu. 